\title{
Azimuthal Reorientation of Pentacene upon 2D Condensation
}

\author{
L. D. Sun, ${ }^{1, *}$ J. Gall, ${ }^{1}$ G. Weidlinger, ${ }^{1}$ C. Y. Liu, ${ }^{2}$ M. Denk, ${ }^{1}$ and P. Zeppenfeld ${ }^{1}$ \\ ${ }^{1}$ Institute of Experimental Physics, Johannes Kepler University Linz, Altenbergerstrasse 69, A-4040 Linz, Austria \\ ${ }^{2}$ State Key Laboratory of Precision Measuring Technology and Instruments, Tianjin University, \\ Weijin Road, CN-300072 Tianjin, China
}

(Received 18 October 2012; revised manuscript received 20 December 2012; published 6 March 2013)

We report a novel two-dimensional gas-solid phase transition of pentacene molecules on the $\mathrm{Cu}(110)-(2 \times 1) \mathrm{O}$ surface where the $2 \mathrm{D}$ condensation is accompanied by a reversible azimuthal rotation of the pentacene molecules. The change of the optical anisotropy associated with this reorientation allows us to explore the $2 \mathrm{D}$ condensation as a function of coverage and temperature by reflectance difference spectroscopy. As a result, the $2 \mathrm{D}$ heat of condensation of pentacene on $\mathrm{Cu}(110)-(2 \times 1) \mathrm{O}$ is determined to be $84 \mathrm{meV}$, which is more than one order of magnitude smaller than the respective value for $3 \mathrm{D}$ crystallization.

DOI: 10.1103/PhysRevLett.110.106101

Adsorption of large $\pi$-conjugated molecules on inorganic solid surfaces is a topic of great interest in both fundamental research and technological applications [1-3]. On the one hand, the morphology and the associated electronic structure of the organic-inorganic interface determine the performance of optoelectronic devices based on organic thin films such as organic field effect transistors, organic light emitting diodes, and organic photovoltaic cells [4]. On the other hand, the large footprint and the numerous internal degrees of freedom of large $\pi$-conjugated molecules may lead to a more complex structural phase diagram as compared to atoms or small molecules [5]. Recent experimental and theoretical studies demonstrate that the conformation, orientation, and deformation of large $\pi$-conjugated molecules can have a sensitive influence on the delicate balance between intermolecular and moleculesubstrate interactions and thus, lead to novel types of structures and phase transitions [6-12].

In this Letter, we report on the adsorption of pentacene, a prototype rod-shaped $\pi$-conjugated molecule, on the highly corrugated, anisotropic $\mathrm{Cu}(110)-(2 \times 1) \mathrm{O}$ surface. We find that pentacene molecules experience a weak attractive intermolecular interaction leading to a fully reversible two-dimensional (2D) gas-solid phase transition upon variation of surface coverage or substrate temperature. A close inspection of this phase transition reveals that the ideal 2D gas law is still valid in spite of the large size and the complex internal structure of pentacene. Moreover, we find that the rod-shaped pentacene molecules reorient themselves upon 2D condensation, thus revealing the anisotropic nature of the intermolecular interaction and its

Published by the American Physical Society under the terms of the Creative Commons Attribution 3.0 License. Further distribution of this work must maintain attribution to the author(s) and the published article's title, journal citation, and DOI.
PACS numbers: 68.35.Rh, 68.43.- h, 78.40.-q, 78.66.Qn

dependence on the azimuthal orientation of the molecules relative to the highly corrugated surface with twofold rotational symmetry.

Reflectance difference spectroscopy (RDS), also known as reflectance anisotropy spectroscopy, measures the difference of the reflectance at normal incidence for two mutually perpendicular orientations of the polarization vector as a function of photon energy [13]. For large $\pi$-conjugated organic molecules, Frederick et al. have demonstrated that RDS is particularly sensitive to the azimuthal molecular orientation on surfaces [14]. In the current study, RDS was applied to investigate the orientational ordering of pentacene as a function of surface coverage $\theta$ and substrate temperature $T_{s}$.

The experiments were carried out in a UHV chamber with a base pressure of $1 \times 10^{-10} \mathrm{mbar}$. The substrate can be heated by electron beam bombardment from the backside and cooled down to $15 \mathrm{~K}$ with a liquid He cryostat. $\mathrm{The} \mathrm{Cu}(110)$ surface was cleaned by repeated cycles of $\mathrm{Ar}^{+}$ion sputtering and subsequent annealing at $800 \mathrm{~K}$. The $\mathrm{Cu}(110)-(2 \times 1) \mathrm{O}$ surface was prepared by exposing the freshly cleaned $\mathrm{Cu}(110)$ surface to more than $20 \mathrm{~L}$ of oxygen $\left(1 \mathrm{~L}=1 \times 10^{-6}\right.$ Torr $\left.\cdot \mathrm{s}\right)$ and subsequent annealing at $600 \mathrm{~K}$. Pentacene molecules were evaporated from a thoroughly degassed Knudsen cell while the sample was held at selected temperatures. The molecular adsorption and the 2D phase transition were monitored in situ using a photoelastic modulator based reflectance difference (RD) spectrometer $[15,16]$. The normalized difference in reflectivity $\Delta R / R=2\left(R_{[1 \overline{1} 0]}-R_{[001]}\right) /\left(R_{[1 \overline{1} 0]}+R_{[001]}\right)$ [17] is recorded either in spectroscopic mode (spanning a photon energy range from 1.5 to $5.5 \mathrm{eV}$ ) or by recording transients, where the $\Delta R / R$ signal at a selected photon energy is monitored as a function of time. In addition, the structure and morphology of the pentacene adlayer was investigated using a homemade variable temperature scanning tunneling microscope and low energy electron 

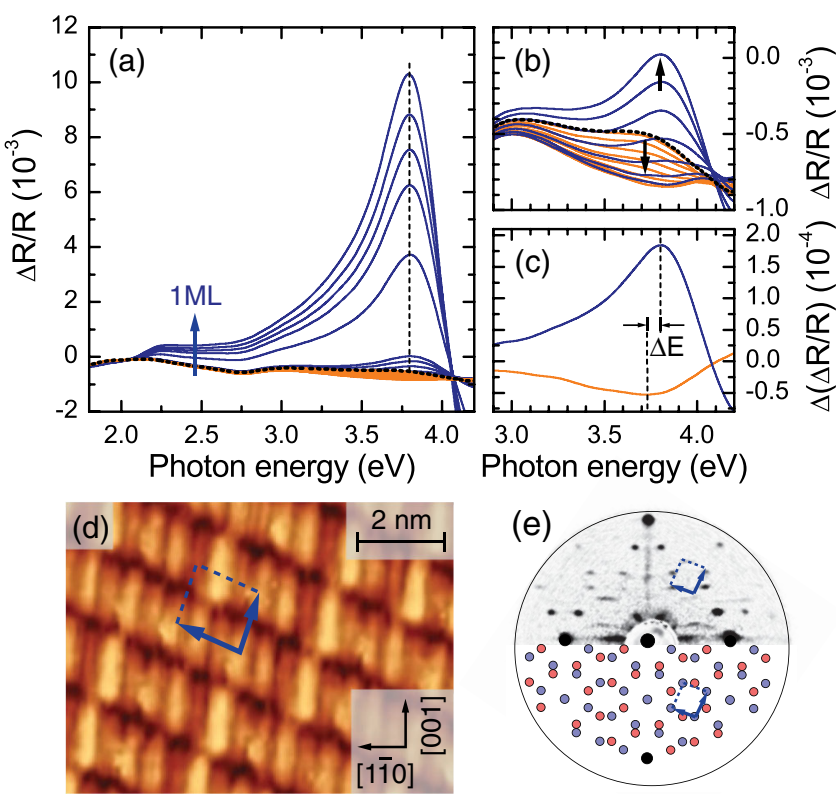

FIG. 1 (color online). (a) RD spectra $(\Delta R / R)$ of the pristine $\mathrm{Cu}(110)-(2 \times 1) \mathrm{O}$ surface (thick black dotted line) and after subsequent deposition of pentacene (thin solid lines): for the first 15 spectra, the coverage increment is $0.013 \mathrm{ML} /$ spectrum up to a total coverage of $0.2 \mathrm{ML}$; the last five spectra correspond to pentacene coverages of $0.5,0.7,0.8,0.9$, and $1 \mathrm{ML}$, respectively. (b) Spectral evolution during the first 15 deposition steps in the energy range around the main peak position. The first 8 spectra growing in negative direction are plotted in orange (light), whereas the rest, growing in positive direction, is plotted in blue (dark). (c) Difference between two RD spectra $[\Delta(\Delta R / R)]$ : 0.1 ML-0.0 ML (orange or light); 0.2 ML-0.1 ML (blue or dark). (d) STM image at $15 \mathrm{~K}$ of a closed pentacene adlayer (1 ML) on $\mathrm{Cu}(110)-(2 \times 1) \mathrm{O}$, deposited at room temperature. The $(14,-32)$ unit cell of the superstructure is indicated. Tunneling current: $20 \mathrm{pA}$; tip bias: $+2 \mathrm{~V}$. (e) LEED pattern of the full pentacene monolayer at $115 \mathrm{~K}$ (upper half) and simulated reciprocal lattice (lower half) with indication of the unit cell of the superstructure.

diffraction (LEED) equipped with a double channel plate detector.

Figure 1(a) shows RD spectra $(\Delta R / R)$ recorded after consecutive deposition of pentacene on $\mathrm{Cu}(110)-(2 \times 1) \mathrm{O}$ at a substrate temperature of $300 \mathrm{~K}$ up to a total coverage of 1 monolayer (ML) (which corresponds to a close-packed layer of lying pentacene molecules). In order to follow the detailed evolution of the molecular orientation during the initial stage of growth, the amount of pentacene deposited in each of the first 15 steps was chosen to be as small as $0.013 \mathrm{ML}$. The RD spectrum corresponding to $1 \mathrm{ML}$ of pentacene exhibits a pronounced peak at around $3.8 \mathrm{eV}$ which can be attributed to the intramolecular electronic transition from the highest occupied molecular orbital (HOMO) to the third lowest unoccupied molecular orbital $($ LUMO + 2) of pentacene [18,19]. Noting that the dipole moment of this transition is oriented along the long molecular axis, the positive sign of the corresponding RD response indicates that the pentacene molecules are lying flat on the surface with their long molecular axis oriented preferentially along the [001] direction of the $\mathrm{Cu}$ substrate (i.e., the direction of the $\mathrm{Cu}-\mathrm{O}$ rows). The morphology resolved by STM [Fig. 1(d)] and LEED [Fig. 1(e)] shows that the pentacene monolayer forms a $\left(\begin{array}{cc}1 & \pm 4 \\ -3 & \pm 2\end{array}\right)$ superstructure with three molecules per unit cell [Fig. 1(d)]. The long molecular axis is, indeed, found to be exclusively aligned along the [001] direction of the substrate. However, a closer inspection of the first $15 \mathrm{RD}$ spectra, recorded at the beginning of the pentacene deposition [Fig. 1(b)], reveals that the $\mathrm{RD}$ signal at $3.9 \mathrm{eV}$, at first, gradually grows in the opposite (negative) direction until a coverage of $\theta_{c} \approx 0.1 \mathrm{ML}$ is reached. In Fig. 1(c), the difference between subsequent RD spectra $[\Delta(\Delta R / R)]$, i.e., the incremental change of the RD spectrum, is shown to illustrate the characteristic difference between the spectra of the pentacene molecules below and above the coverage $\theta_{c}$, respectively: the orange (light) curve corresponds to the change of $\Delta R / R$ from zero coverage up to $\theta_{c}$, whereas the blue (dark) curve depicts the change over the coverage range from $\theta_{c}$ to $2 \theta_{c}$. The two curves clearly differ with respect to sign, line shape, and amplitude. Also a slight shift of the peak position can be discerned. This observation unambiguously shows that pentacene adsorbs in two distinct phases with different molecular alignment and different electronic structure below and above $\theta_{c}$. Compared to the characteristic spectrum of the high coverage (well-ordered) phase, the negative, broadened line shape with smaller amplitude observed at $\theta<\theta_{c}$ indicates a much lower degree of orientational ordering, but with net molecular orientation along the [110] direction of the substrate [20]. From these experimental observations we infer the following growth scenario: at low coverages, pentacene molecules adsorb in a 2D gas phase, which then condenses into a $2 \mathrm{D}$ solid phase at the coverage $\theta_{c}$. Such 2D phase transitions are well-known for atoms and small molecules and are typical for adsorbates with attractive lateral interactions [21,22].

Figures 2(a) and 2(b) show RD transients at a photon energy of $3.9 \mathrm{eV}$, and the spectral evolution, respectively, for $0.25 \mathrm{ML}$ pentacene on $\mathrm{Cu}(110)-(2 \times 1) \mathrm{O}$ as a function of substrate temperature. The fully reversible temperature dependence of the RD signal at $3.9 \mathrm{eV}$ means that at all times 2D condensed phase and 2D gas phase are in thermal equilibrium, which, in turn proves that this is indeed an entropy-driven equilibrium phase transition. The corresponding morphology has also been studied by STM. Figures 2(c) and 2(d) show STM images of the same sample, namely, $0.2 \mathrm{ML}$ pentacene on $\mathrm{Cu}(110)-(2 \times 1) \mathrm{O}$, recorded at substrate temperatures of 15 and $300 \mathrm{~K}$, respectively. While we were able to achieve atomic resolution of the $\mathrm{Cu}(110)-(2 \times 1) \mathrm{O}$ surface structure, well ordered pentacene islands could only be found on the surface at low 


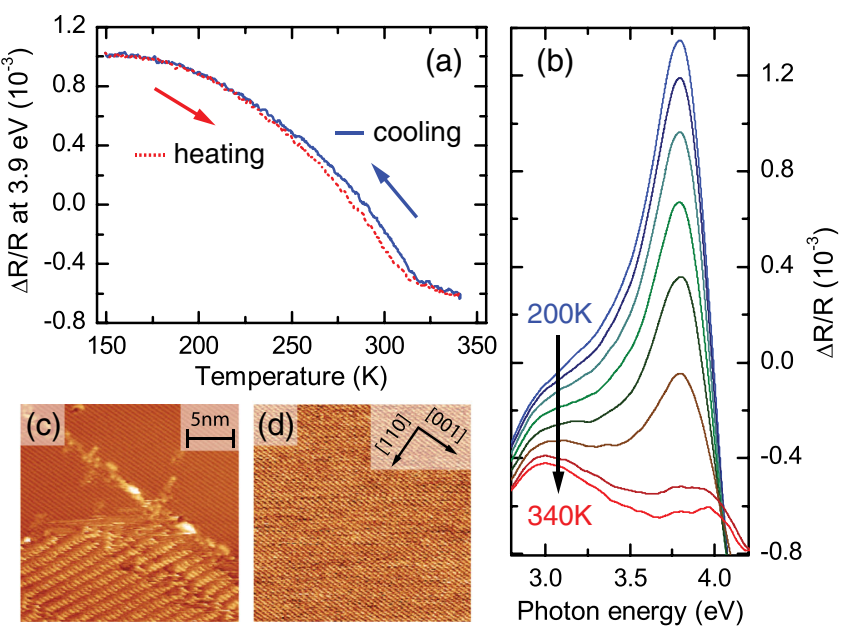

FIG. 2 (color online). (a) $\Delta R / R$ signal at $3.9 \mathrm{eV}$ recorded during heating and cooling of a $0.25 \mathrm{ML}$ pentacene adlayer on $\mathrm{Cu}(110)-(2 \times 1) \mathrm{O}$; heating rate: $0.2 \mathrm{~K} / \mathrm{s}$. (b) Corresponding RD spectra recorded at selected temperatures during heating or cooling $(200 \mathrm{~K}, 220 \mathrm{~K}, 240 \mathrm{~K}, 260 \mathrm{~K}, 280 \mathrm{~K}, 300 \mathrm{~K}, 320 \mathrm{~K}$, and $340 \mathrm{~K}$, from top to bottom). (c) STM image at $15 \mathrm{~K}$ of a $0.20 \mathrm{ML}$ pentacene adlayer on $\mathrm{Cu}(110)-(2 \times 1) \mathrm{O}$. (d) $\mathrm{STM}$ image at $300 \mathrm{~K}$ of the same sample as shown in (c). Tunneling current: 20 pA; tip bias: $+2 \mathrm{~V}$.

temperatures. At room temperature, only fuzzy features, most likely induced by pentacene molecules moving under the STM tip, were observed. Evidently, the STM study confirms that, at low surface coverage, pentacene molecules condense into 2D islands at low temperature but form a highly mobile 2D gas phase at room temperature.

Furthermore, we have determined the onset of condensation (i.e., the coverage $\theta_{c}$ ) as a function of substrate temperature. Figure 3(b) shows the evolution of the $\Delta R / R$ signal at $3.9 \mathrm{eV}$ and a substrate temperature of $250 \mathrm{~K}$ as a function of pentacene coverage $\theta$ (adsorption
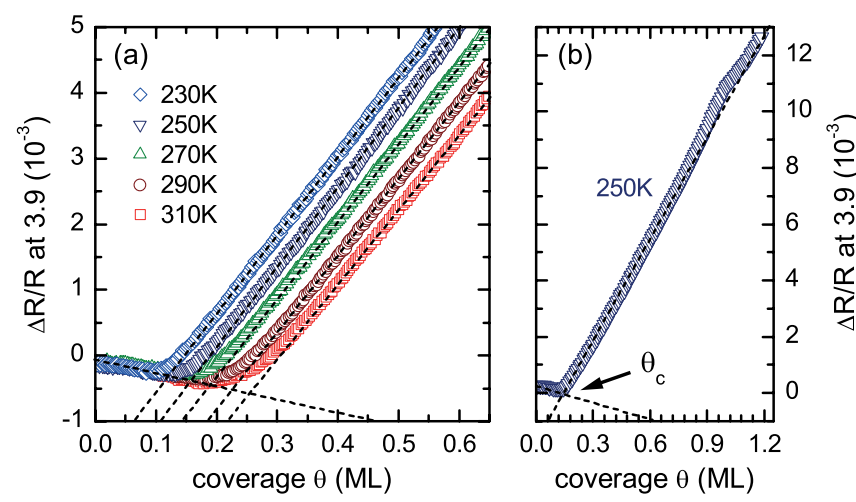

FIG. 3 (color online). RD signal intensity $(\Delta R / R)$ at a photon energy of $3.9 \mathrm{eV}$, recorded during the deposition of pentacene on $\mathrm{Cu}(110)-(2 \times 1) \mathrm{O}$ : (a) initial stage of growth (up to $0.6 \mathrm{ML}$ ) for five different substrate temperatures $(230 \mathrm{~K}, 250 \mathrm{~K}, 270 \mathrm{~K}$, $290 \mathrm{~K}$, and $310 \mathrm{~K}$, from left to right, respectively). (b) Complete monolayer growth curve at a substrate temperature of $250 \mathrm{~K}$. isotherm): a slow, negative slope of the RD transient at the beginning of pentacene deposition is followed by a fast increase with positive slope. The slight increase in slope just before the completion of the first monolayer could be due to an enhanced condensation rate or to slight changes in the structure or conformation in the condensed phase upon 2D compression. We are able to explain the curve shape of these adsorption isotherms according to the discussion above: the negative slope at the initial stage of growth is due to the formation of a $2 \mathrm{D}$ gas phase. The change in sign at a coverage of around 0.1 ML marks the surface coverage $\left(\theta_{c}\right)$ at which the condensation of the 2D gas phase into a 2D solid phase sets in. The subsequent linear increase of the RD signal directly correlates with the increase of the coverage of the 2D solid phase. The presence of a condensation point implies a net attractive interaction between pentacene molecules adsorbed on the surface. By studying the dependence of the onset of condensation $\theta_{c}$ on the substrate temperature $T_{s}$, the 2D heat of condensation $\Delta H$ can be deduced from the 2D ClausiusClapeyron equation [23]

$$
\frac{d\left(\ln \varphi_{c}\right)}{d\left(1 / T_{s}\right)}=\frac{\Delta H}{k_{B}},
$$

where $\varphi_{c}$ is the 2D spreading pressure, which can be related to the coverage $\theta_{c}$ through the ideal gas law, namely, $\varphi_{c}=n_{s} \theta_{c} k_{B} T_{s}$ (with $n_{s}$ being the surface density of the substrate atoms and $k_{B}$ the Boltzmann constant). From the dependence of $\theta_{c}$ on the substrate temperature $T_{s}$, extracted from Fig. 3(a), $\Delta H$ can be deduced directly by plotting the logarithm of $\varphi_{c}$ as a function of the inverse temperature $T_{s}^{-1}$ (Fig. 4). The slope of the linear fit to the data points (solid red line in Fig. 4) yields a 2D heat of condensation of $\Delta H=84 \pm 2 \mathrm{meV}$ per pentacene molecule. The fact that the experimental data points lie on a straight line corroborates the validity of our assumptions, in particular the appropriateness to describe the low density gas phase by the ideal gas law. The 2D heat of condensation $\Delta H$ determined in this work is more than one order of

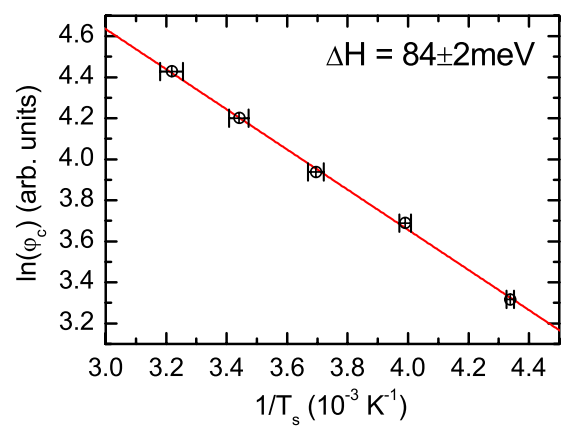

FIG. 4 (color online). Logarithm of the 2D spreading pressure $\varphi_{c}\left(\ln \theta_{c} T_{s}\right)$ as a function of the inverse temperature $\left(1 / k_{B} T_{s}\right)$. The data points were deduced from the onset of condensation (coverages $\theta_{c}$ in Fig. 3) and fitted by a linear curve (red solid line). 
magnitude smaller than that of the 3D pentacene crystal $(1.58 \mathrm{eV})$, in which van der Waals intermolecular interactions play the key role $[24,25]$. The significant reduction of the 2D condensation energy of pentacene on $\mathrm{Cu}(110)-(2 \times 1) \mathrm{O}$, with respect to the $3 \mathrm{D}$ crystal, could be due to several reasons. (i) First of all, the effective coordination of the molecules in the $2 \mathrm{D}$ condensed layer is reduced with respect to the $3 \mathrm{D}$ bulk. Moreover, the intermolecular interactions are anisotropic. Therefore, the intermolecular binding energy also depends on the relative orientation of the molecules and their mutual arrangement as well as on the dimensionality of the packing. (ii) The 2D gas phase may be composed of small pentacene aggregates (e.g., dimers) in which molecules are already bound to each other. Upon condensation of such aggregates, this internal binding energy would not be included in the 2D condensation enthalpy $\Delta H$ as determined from the data in Fig. 4. (iii) The molecule-substrate interaction, which is completely absent in the case of the 3D pentacene crystal, significantly alters the nature and strength of the intermolecular interaction between adsorbed molecules. In the context of substrate-mediated interactions, it is also interesting to compare the results of the current study with pentacene adsorption on bare $\mathrm{Cu}(110)$ [26,27]. The molecule-substrate interaction is substantially stronger for pentacene on $\mathrm{Cu}(110)$ as compared to $\mathrm{Cu}(110)-(2 \times 1) \mathrm{O}$. This is evidenced by the following observations: (i) a strong hybridization reflected by a measurable charge transfer from the substrate to the molecules has been observed for pentacene adsorbed on bare $\mathrm{Cu}(110)$ [28], but not for pentacene on $\mathrm{Cu}(110)-(2 \times 1) \mathrm{O}$ [29], and (ii) the desorption temperature of pentacene molecules is much higher on $\mathrm{Cu}(110)$ than on $\mathrm{Cu}(110)-(2 \times 1) \mathrm{O}(T>$ $800 \mathrm{~K}$ vs $T \approx 430 \mathrm{~K}$ ) [30,31]. This significant reduction of molecule-substrate interaction can be assigned to the passivation of the $\mathrm{Cu}(110)$ surface by adsorption of oxygen. The reportedly repulsive interaction of pentacene molecules adsorbed on the bare $\mathrm{Cu}(110)$ surface could be due to a sizable dipole moment induced by the strong electronic hybridization, whereas the weaker interaction between pentacene and the $\mathrm{Cu}(110)-(2 \times 1) \mathrm{O}$ surface leads to a smaller repulsive component to the effective lateral interaction, which may be completely outweighed by the van der Waals force, thus resulting in a weak, but still attractive, net intermolecular interaction. In fact, the reorientation of pentacene molecules upon 2D condensation, as revealed in the present study, prominently attests the importance of this delicate balance between competing intermolecular and molecule-substrate interactions.

In conclusion, by monitoring the evolution of the in-plane optical anisotropy, we have been able to reveal the $2 \mathrm{D}$ gas to solid phase transition of pentacene molecules adsorbed on $\mathrm{Cu}(110)-(2 \times 1) \mathrm{O}$. This phase transition is driven by a small condensation enthalpy of $\Delta H=$ $84 \pm 2 \mathrm{meV}$. Most remarkably, this phase transition is accompanied by an azimuthal reorientation of the molecules which can be interpreted by two intercorrelated effects: the dependence of the intermolecular interactions on the azimuthal orientation of the molecules, and the dependence of the molecular alignment on the lateral bonding conditions. Those are essentially two aspects of a single physical effect, namely, the competition between intermolecular and molecule-substrate interactions of molecules adsorbed on a surface.

We acknowledge financial support for this work by the Austrian Science Fund FWF under Project No. P21422.

*lidong.sun@jku.at

[1] S. R. Forrest, Chem. Rev. 97, 1793 (1997).

[2] F. Schreiber, Prog. Surf. Sci. 65, 151 (2000).

[3] J. V. Barth, Annu. Rev. Phys. Chem. 58, 375 (2007).

[4] Physical and Chemical Aspects of Organic Electronics, edited by C. Wöll (Wiley-VCH, Weinheim, 2009).

[5] S. M. Barlow and R. Raval, Surf. Sci. Rep. 50, 201 (2003).

[6] A. Hauschild, K. Karki, B. C. C. Cowie, M. Rohlfing, F. S. Tautz, and M. Sokolowski, Phys. Rev. Lett. 94, 036106 (2005).

[7] R. Temirov, S. Soubatch, A. Luican, and F. S. Tautz, Nature (London) 444, 350 (2006).

[8] L. Kilian, A. Hauschild, R. Temirov, S. Soubatch, A. Schöll, A. Bendounan, F. Reinert, T.-L. Lee, F. S. Tautz, M. Sokolowski et al., Phys. Rev. Lett. 100, 136103 (2008).

[9] C. Stadler, S. Hansen, I. Kröger, C. Kumpf, and E. Umbach, Nat. Phys. 5, 153 (2009).

[10] B. Bröker, O. T. Hofmann, G. M. Rangger, P. Frank, R.-P. Blum, R. Rieger, L. Venema, A. Vollmer, K. Müllen, J.P. Rabe et al., Phys. Rev. Lett. 104, 246805 (2010).

[11] A. Schöll, L. Kilian, Y. Zou, J. Ziroff, S. Hame, F. Reinert, E. Umbach, and R. H. Fink, Science 329, 303 (2010).

[12] V. Lanzilotto, C. Sanchez-Sanchez, G. Bavdek, D. Cvetko, M. F. Lopez, J. A. Martin-Gago, and L. Floreano, J. Phys. Chem. C 115, 4664 (2011).

[13] P. Weightman, D. S. Martin, R. J. Cole, and T. Farrell, Rep. Prog. Phys. 68, 1251 (2005).

[14] B. G. Frederick, J. R. Power, R. J. Cole, C. C. Perry, Q. Chen, S. Haq, T. Bertrams, N. V. Richardson, and P. Weightman, Phys. Rev. Lett. 80, 4490 (1998).

[15] D. E. Aspnes, J. P. Harbison, A. A. Studna, and L. T. Florez, J. Vac. Sci. Technol. A 6, 1327 (1988).

[16] O. Acher and B. Drévillon, Rev. Sci. Instrum. 63, 5332 (1992).

[17] C. Goletti, G. Bussetti, P. Chiaradia, A. Sassella, and A. Borghesi, Org. Electron. 5, 73 (2004).

[18] T. M. Halasinski, D. M. Hudgins, F. Salama, L. J. Allamandola, and T. Bally, J. Phys. Chem. A 104, 7484 (2000).

[19] P. Sony and A. Shukla, Phys. Rev. B 75, 155208 (2007).

[20] T. Dienel, C. Loppacher, S. C. B. Mannsfeld, R. Forker, and T. Fritz, Adv. Mater. 20, 959 (2008).

[21] B. Poelsema, L. K. Verheij, and G. Comsa, Phys. Rev. Lett. 51, 2410 (1983).

[22] A. Marmier, C. Ramseyer, P. N. M. Hoang, C. Girardet, J. Goerge, P. Zeppenfeld, M. Büchel, R. David, and G. Comsa, Surf. Sci. 383, 321 (1997). 
[23] T. Hill, J. Chem. Phys. 17, 520 (1949).

[24] J. S. Chickos and W.E. Acree, J. Phys. Chem. Ref. Data 31, 537 (2002).

[25] D. Nabok, P. Puschnig, and C. Ambrosch-Draxl, Phys. Rev. B 77, 245316 (2008).

[26] S. Lukas, G. Witte, and C. Wöll, Phys. Rev. Lett. 88, 028301 (2001).

[27] K. Müller, A. Kara, T. K. Kim, R. Bertschinger, A. Scheybal, J. Osterwalder, and T. A. Jung, Phys. Rev. B 79, 245421 (2009).
[28] H. Yamane, D. Yoshimura, E. Kawabe, R. Sumii, K. Kanai, Y. Ouchi, N. Ueno, and K. Seki, Phys. Rev. B 76, 165436 (2007).

[29] S. Berkebile, G. Koller, A. J. Fleming, P. Puschnig, C. Ambrosch-Draxl, K. Emtsev, T. Seyller, J. Riley, and M. G. Ramsey, J. Electron Spectrosc. Relat. Phenom. 174, 22 (2009).

[30] S. Söhnchen, S. Lukas, and G. Witte, J. Chem. Phys. 121, 525 (2004).

[31] J. Gall, L. D. Sun, and P. Zeppenfeld (unpublished). 